\title{
Comment on "Pygmy dipole response of proton-rich argon nuclei in random-phase approximation and no-core shell model"
}

\author{
N. Paar* \\ Physics Department, Faculty of Science, University of Zagreb, Croatia
}

(Dated: November 1, 2018)

In a recent article by C. Barbieri, E. Caurier, K. Langanke, and G. Martínez-Pinedo [1], low-energy dipole excitations were studied in proton-rich ${ }^{32,34} \mathrm{Ar}$ with random-phase approximation (RPA) and no-core shell model (NCSM) using correlated realistic nucleon-nucleon interactions obtained by the unitary correlation operator method (UCOM) 2]. The main objective of this Comment is to argue that the article [1] contains an inconsistency with respect to previous study of excitations in the same UCOM-RPA framework using identical correlated Argonne V18 interaction [3], it does not provide any evidence that the low-lying state declared as pygmy dipole resonance in ${ }^{32} \mathrm{Ar}$ indeed has the resonance-like structure, and that prior to studying exotic modes of excitation away from the valley of stability one should ensure that the model provides reliable description of available experimental data on nuclear ground state properties and excitations in nuclei. Although the authors aimed at testing the UCOM based theory at the proton drip line, available experimental data that are used as standard initial tests of theory frameworks at the proton drip line have not been considered in the UCOM case (e.g., binding energies, one-proton separation energies, two-proton separation energies).

The parametrized correlation functions that describe the short-range correlations in the UCOM framework have been constrained at the level of the two-nucleon system, and their ranges are determined by fitting the NCSM binding energies of ${ }^{3} \mathrm{H}$ and ${ }^{4} \mathrm{He}$ to the experimental values [4]. In this way, some of the missing ingredients (e.g., the three-body force, higher orders in cluster expansion) are effectively taken into account and included in the parameters of correlation functions. Despite the fact that the short-range properties of the correlated realistic nucleon-nucleon interaction are well behaved and some of the three-body effects are implicitly included, the UCOM framework cannot provide quantitative description of nuclear structure properties within Hartree-Fock (HF) and Fermionic molecular dynamics (FMD). Therein the binding energies of nuclei across the nuclide chart are dramatically underestimated (in $\mathrm{HF}$ up to $50 \%$ ), and the radii are smaller by 1-3 fm when compared to experimental data [5]. A considerable portion of the missing correlations can be recovered, e.g., by many-body perturbation theory on top of HF [5], or by introducing additional purely phenomenological terms with new free parameters

\footnotetext{
*npaar@phy.hr
}

supplemented to the correlated realistic nucleon-nucleon interaction (e.g. in FMD [6]).

In Ref. 3] the UCOM HF + RPA based on correlated Argonne V18 interaction is introduced and tested on several nuclei from ${ }^{16} \mathrm{O}$ toward ${ }^{208} \mathrm{~Pb}$. It has been pointed out that due to unrealistic descriptions of the HF ground state (e.g., single-particle spectra are extremely wide), the missing long-range correlations and three-body interaction, the energies of giant resonances are strongly overestimated in comparison to experimental data and previous studies. In particular, excitation energies of isovector giant dipole resonances (IVGDR) are $\approx 3-8 \mathrm{MeV}$ higher than those of the experimental values [3]. Despite these facts, C. Barbieri, E. Caurier, K. Langanke, and G. Martínez-Pinedo employ the same UCOM HF + RPA based on identical correlated Argonne V18 interaction in the study of an exotic excitation in the $1^{-}$channel: proton pygmy dipole resonance (PPDR) in ${ }^{32,34} \mathrm{Ar}[1]$. One should be concerned by the fact that UCOM RPA which seriously overestimates IVGDR across the nuclide chart from ${ }^{16} \mathrm{O}$ toward ${ }^{208} \mathrm{~Pb}[3]$, in the case of Ar isotopes appears in rather good agreement with empirical estimates [1] and relativistic QRPA which on the other hand quantitatively also describe giant resonances in other nuclei [7]. The reported UCOM RPA results for ${ }^{32,34} \mathrm{Ar}$ are obviously inconsistent with previous systematic study based on the same model and effective interaction, employed along the nuclide chart including the region of medium heavy nuclei (Fig. 10 in Ref. [3] ).

Although in Ref. 1] Barbieri et al. claim that a clear peak associated with pygmy dipole resonance has been found in UCOM RPA and NCSM calculations in ${ }^{32} \mathrm{Ar}$, they do not provide any supporting evidence on the resonance-like structure of the corresponding state. Based on transition densities only, one cannot draw conclusions on the collectivity of the low-energy excitations; i.e., the same transition densities as presented in Ref. [1] in the upper panel of Fig. 3, could also have the origin only in single-particle transitions [8]. In Ref. 7] it has been pointed out that actually the pairing correlations play an essential role in building up the collectivity of the PPDR mode in ${ }^{32} \mathrm{Ar}$ and other medium heavy nuclei close to the proton drip line. This means that the structure of a single relevant low-energy state becomes more distributed; i.e., a considerable number of two-quasiparticle configurations contribute to a particular excitation mode. In the limiting case when pairing correlations are absent, the collectivity of the low-lying states vanishes; i.e., each low-lying state is dominated mainly by a single-particle transition. In the article by Barbieri et al. the pairing 
correlations are not properly included in $\mathrm{HF}+\mathrm{RPA}$ calculations (partial occupation of $\pi 0 d_{3 / 2}$ orbit is set to $1 / 2$, other orbits are fully occupied or empty; there is no pairing interaction in ground state calculations and residual RPA interaction). In contrast, shell model calculations, performed in the full $0 \hbar \omega$ space, include pairing with all the possible seniorities. Unfortunately, it remains unknown whether there is some difference in the nature of the observed low-energy excitations in HF + RPA and shell model calculations. Although the issue of collectivity in the low-lying dipole states is simply ignored, the term "pygmy dipole resonance" has extensively been used throughout the article [1].

Recent study of giant resonances using correlated realistic nucleon-nucleon interactions which include couplings with complex configurations $(2 \mathrm{p} 2 \mathrm{~h})$, indicates that actually the UCOM RPA response of giant resonances considerably shifts toward lower energies [9]. In the case of dipole excitations in ${ }^{16} \mathrm{O}$ and ${ }^{40} \mathrm{Ca}$, the transition strengths are systematically lowered by $\approx 8 \mathrm{MeV}$. The same effect should also appear in the case of ${ }^{32} \mathrm{Ar}$; i.e., one could expect that the UCOM RPA centroid energy of low-lying states will be shifted from the value of $9.15 \mathrm{MeV}$ reported in Ref. [1] to significantly lower values. It remains a puzzle why the NCSM calculations for ${ }^{32,34} \mathrm{Ar}$ [1] result in strength distributions very similar to those of UCOM RPA (the centroid energies are different by less than $1 \mathrm{MeV}$ ), although the former includes up to $2 \mathrm{p} 2 \mathrm{~h}$ configuration spaces. Prior to implementation of UCOM NCSM on excitations in exotic nuclei, the effect of the $2 \mathrm{p} 2 \mathrm{~h}$ model space should have carefully been checked on stable nuclei (e.g. ${ }^{16} \mathrm{O},{ }^{40} \mathrm{Ca},{ }^{48} \mathrm{Ca}$, etc.) where extensive experimental data on giant resonances already exist, as has been done, e.g., in the UCOM second-RPA study [9].
Finally, planned experiments aimed at observing the PPDR mode in Ar isotopes do not present the best choice for testing the accuracy of UCOM RPA and NCSM approaches. Prior to studying exotic modes of excitation, theory models should be tested on the large set of already existing experimental data on the nuclear ground state and excitation properties in nuclei. In addition, there is a whole set of data available on the properties of nuclei at proton drip line (e.g., binding energies, one-proton separation energies, two-proton separation energies) that should be used for testing the accuracy of UCOM based approaches at the proton drip line before studying excitations. Finally, there is a considerable amount of data on giant resonances in stable nuclei, as well as recent data on pygmy dipole resonances in the unstable nucleus ${ }^{132} \mathrm{Sn}$ [10]. All these data have systematically been used in numerous studies and should be used in the first place to validate the implementation of effective interactions in nuclear many-body theories. Over the past decades, much effort has been expended to achieve the microscopic description of nuclear ground state and excitations, and available experimental data provide a crucial test of nuclear structure models. These essential studies should not be bypassed in the case of UCOM based theory if the same approach aims at description of exotic modes of excitation in nuclei away from the valley of stability.

\section{Acknowledgments}

This work was supported by the Unity through Knowledge Fund (UKF Grant No. 17/08).
[1] C. Barbieri, E. Caurier, K. Langanke, and G. MartínezPinedo, Phys. Rev. C 77, 024304 (2008).

[2] H. Feldmeier, T. Neff, R. Roth, and J. Schnack, Nucl. Phys. A 632, 61 (1998).

[3] N. Paar, P. Papakonstantinou, H. Hergert, and R. Roth, Phys. Rev. C 74, 014318 (2006).

[4] R. Roth, H. Hergert, N. Paar, and P. Papakonstantinou, Nucl. Phys. A 788, 12c (2007).

[5] R. Roth, P. Papakonstantinou, N. Paar, H. Hergert, T. Neff, and H. Feldmeier, Phys. Rev. C 73, 044312 (2006).

[6] M. Chernykh, H. Feldmeier, T. Neff, P. von Neumann-
Cosel, and A. Richter, Phys. Rev. Lett. 98, 032501 (2007).

[7] N. Paar, D. Vretenar, and P. Ring, Phys. Rev. Lett. 94, 182501 (2005).

[8] D. Vretenar, N. Paar, P. Ring, and G. A. Lalazissis, Nucl. Phys. A 692, 496 (2001).

[9] P. Papakonstantinou and R. Roth, arXiv:0709.3167, submitted to Phys. Lett. B (2008).

[10] P. Adrich et al., Phys. Rev. Lett. 95, 132501 (2005). 